screening tests. Serum specimens from eight probable Group $\mathrm{O}$ infections from Cameroon were tested with $10 \mathrm{HIV}$ antibody screening test assays licensed by the U.S. Food and Drug Administration (FDA). All FDA assays based on synthetic peptides or recombinant antigens failed to detect at least one of the infections; assays based on whole-virus lysates performed better.

The test used most commonly in U.S. blood banks failed to detect HIV-1 antibodies in two specimens. These findings ate consistent with other reports on the sensitivity of European HIV antibody tests for group O infections. However, the researchers caution that these tests should not be abandoned hastily because use of these antigens and formats has resulted in significant advances in assay sensitivity and specificity for the subtypes of HIV-1 commonly found in the United States and Europe. Modifications to increase sensitivity for group $\mathrm{O}$ infections needs to be evaluated to ensure that addition of group $\mathrm{O}$ antigens does not result in the loss of sensitivity and specificity for antibodies against the more prevalent HIV-1 viruses.

Data from U.S. blood transfusion services indicate that a very small number of blood donors in the U.S. come from countries in which group $\mathrm{O}$ viruses appear endemic. Momover, of the more than 400,000 AIDS cases in the U.S. reported through August 1994, only 13 represented persons who reported Cameroon, Gabon, or the Central African Republic as their country of origin.

Nevertheless, the potential for divergent strains to infect persons living in the U.S., and, in some cases, to remain undetected by current HIV antibody tests, is of concern for public health and blood-banking communities. The researchers note that active surveillance for variants of HIV-1 and HIV-2 will be important to evaluate the sensitivity of HIV screening tests for detecting these variants as they are identified and to modify tests judiciously to correct for deficiencies in sensitivity

FROM: Schable C, Zekeng L, Pau C, et al. Sensitivity of United States HIV antibody tests for detection of HIV-1 group O infections. Lancet November 12, 1994;344:1333-1334.

\section{New Publication Focuses on Healthcare Worker Safety}

A dvances in Exposure Prevention (A EP) is a new publication that focuses on reducing healthcare workers' risk of occupational infection by blood-borne pathogens. Dr. Janine Jagger of the University of Virginia, editor, is the developer of EPINet (Exposure Prevention Information Network), a software system that assists hospitals in tracking needlestick injuries. AEP will report needlestick injury data regularlymuch of the data will be based on EPINet data. According to Jagger, the research findings presented in AEP will allow hospitals to compare their needlestick injury rates to those of other hospitals. In addition, A EP will report results of field trials of new devices, actions of federal agencies regarding workplace regulations, new device approvals, and new data on occupationally acquired infections. For information, call (804) 982-3763 or FAX (804) 982-0821.

\section{AHA Asks Joint Commission to Clarify Role}

The American Hospital Association (AHA) recently called for the Joint Commission on Accreditation of Healthcare Organizations to develop a strategic plan to clarify its role as the primary accrediting body for U.S. hospitals and healthcare facilities. Affirming its strong support for public disclosure of hospital-specific quality information, the AHA nonetheless pointed out the urgent need to address the inconsistency and variability in the surveys, surveyors, and educational programs to ensure the quality and value of the survey process. The AHA call to action was prompted by the fact that 15 to 20 state hospital associations are exploring options to accreditation by the Joint Commission. This action by even a few states could jeopardize the future of accreditation.

AHA Chairman of the Board Carol Roberts said the AI-IA is committed to having effective methods to assure the public about the quality of the nation's hospitals and healthcam systems and supports the Joint Commission as the most appropriate vehicle to achieve this objective. The AI-IA is a sponsoring agency of the Joint Commission along with the American Medical Association, the American Dental Association, the American College of Surgeons, and the American College of Physicians.

FROM: AHA News December 12, 1994.

\section{CDC Responds to Media Reports That Florida Dentist N ot Source of HIV Infection for Six Patients}

It has been 4 years since the results of an investigation of a Florida dentist with AIDS, Dr. David Acer. The investigation concluded that HIV was transmitted from the dentist to six patients, The exact method of transmission has never been determined but has prompted a myriad of theories in both the mass media and scientific publications. For instance, transmission by contaminated instruments has been widely postulated despite lack of evidence, and the "murder theory' was highlighted in a television show in 1993. Most recently, there have been numerous newspaper articles and a "60 Minutes" television segment broadcast in June 1994 that posed the question "What if the dentist didn't do it?"

Dr. Carol Ciesielski of the CDC, primary investigator of the Florida dental cases, addressed some of the misinformation and rumors about the case in a recent issue of the Annals of Internal M edicine.' She pointed out that the media relied heavily on information from depositions related to private litigation generated by the case and on the findings of investigators hired by the dentist's insurance company to find information that could cast doubt on the conclusion that the patients had been infected by the dentist. These findings were used to suggest that each of the six patients had other risk factors for HIV infection and that the results of the HIV DNA sequence analysis are inconclusive. However, Dr. Ciesielski explained that important facts were omitted from the reports that contradict their conclusions. 